\title{
T-cell immunomodulatory protein in the sea star Asterias rubens: Genomic studies
}

\author{
Michel Leclerc* \\ 556 rue Isabelle Romée, 45640 Sandillon, France
}

\begin{abstract}
The transcriptome of the sea star Asterias rubens shows a T-cell immunomodulatory gene when compared to mouse genome. This gene plays a role in the regulation of graft-versus host reaction in mammals and in sea star system.
\end{abstract}

\section{Introduction}

In mammals, the T-cell immunomodulatory protein is a modulator of $\mathrm{T}$-cell function. It has a protective effect in graft-versus-host disease model [1]. We have, in the past, demonstrated the existence of $\mathrm{T}$ and $\mathrm{B}$ sea star lymphocytes [2]. In the present paper we tried to determine proteins bound to sea star T lymphocytes, as the T-cell immunomodulatory protein(TIP).

\section{Material and methods}

Sea stars were obtained from Gothenburgh University(Sweden).

Immunizations, genomic studies were already described [3].

After ligation of adapters for Illumina's GSII sequencing system, the cDNA, was sequenced on the Illumina GSII platform sequencing.1.100 bp from one side of the approximately $200 \mathrm{bp}$ fragments sequences were assembled using Velvet [4].

\section{Results}

$\mathrm{T}$-cell immunomodulatory protein is present in immunized and non-immunized to HRP sea star genomes.

Result with immunized animals is given:

One contig (Contig11337|m.9741) could be annotated via BLASTX to Mus musculus "T-cell immunomodulatory protein OS = Mus musculus" from the Swissprot database (TIP_MOUSE), with an e-value of $2.35 \mathrm{e}-19$. On an aligned region of 113 amino acids, 74 positive and 54 identical amino acids were found.

5'T GCA A C T T C C T T A C A T CA T C C TGGG T C T T GG T C C A A A T C C A A A C T A C G T T G A T T C T T T A A C A G T GA GCA T T GCA GGCGCA A C A A A A C GA GA C A C A C C G A A C A C A A G A C A G C A C A C T T G G A T G T C G A T C A T C C C A A A C T C A G A G C T G A T A G -

Copyright: (C2016 Leclerc M. This is an open-access article distributed under the terms of the Creative Commons Attribution License, which permits unrestricted use, distribution, and reproduction in any medium, provided the original author and source are credited.
C A A T T C C A T A T C C C C C T G A C G A A C C G G A G G A G T G GA C GA G T G T C C T G T T A A T T A C C C C T G G T A G A T C C G T T C T A T T G A C C G G T G G C G T T C T T A T A G G A A C C T G T G T A T T T A T G G C A A T C G T A G T T G G C G T A C T C C A A C T T C T A G A A A A G A GGGAAGATGATCGAGAAAAGAGACAGGAGTCTCACCGATTC3'

\section{Discussion and conclusion}

Similar results were obtained with non-immunized sea star to HRP.

It is noticeable to recall that $\mathrm{T}$ sea star lymphocytes induce a graftversus-host in vertebrates [2].

when injected by the use of sea star T cell suspension: it induces splenomegaly in chicken and angiogenesis in irradiated mice. It would be interesting to perform these experiments in presence of T-cell immunomodulatory protein, at various concentrations, and to observe, the effect of such a protein on chicken and irradiated mice (inhibitor effect or not).

The emergence of T-cell immunomodulatory protein in sea star genome seems fundamental to the physiology of Asterids and constitutes "a biological marker" of these last ones.

\section{References}

1. Fiscella M, Perry JW, Teng B,Bloom M, Zhang C, et al. (2003) TIP, a T-cell factor identified using high-throughput screening increases survival in a graft-versus-host disease model. Nature Biotechnology 21: 302-307. [Crossref]

2. Vincenta N, Osterasa M, Ottena P, Leclerc M (2012) A new gene in A. rubens: A sea star Ig kappa gene. Amer J Immunol 8: 191-195. [Crossref]

3. Leclerc M and Otten P (2013) Evidence of Interleukin Genes In The Sea-Star: Asterias Rubens (Echinoderma) Immunol Lett 15: 68-70.

4. Zerbino DR, Birney E, et al. (2007) Velvet: Algorithms for de novo short read assembly using de Bruijn graphs. Gen Res 18: 821-829. [Crossref]

Correspondence to: Michel Leclerc, 556 rue Isabelle Romée, 45640 Sandillon, France, Tel: 02384102 09; E-mail: mleclerc45@gmail.com

Key words: T-cell immunomodulatory protein, sea star, genomic

Received: April 02, 2016; Accepted: April 22, 2016; Published: April 25, 2016 